\title{
A CORRECT DERIVATION OF EXACT SOLUTION OF DISPERSION MODEL FOR TRACER PULSE IN A BATCH LOOP REACTOR
}

\author{
MASAHARU TAKAO AND YASUHIRO MURAKAMI \\ Department of Chemical Engineering, Kyushu University, Fukuoka 812
}

TSUTOMU HIROSE

Department of Applied Chemistry, Kumamoto University, Kumamoto 860

Key Words: Chemical Reactor, Mixing, Loop Reactor, Dispersion Model, Tracer Pulse, Batch System,

Laplace Transform, Exact Solution

Dear Sir:

The exact form of Eq. $(5)^{1 /}$ has been recently found to be correctly derived by a Laplace transform method. In addition, there is an incorrect expression in the initial condition of Eq. (2). ${ }^{1)}$

We propose a correct expression of the initial condition and briefly a process of solving directly the dispersion model under the boundary conditions of Eqs. (3) and (4) ${ }^{11}$ together with the initial condition corrected.

The initial condition was expressed as

$$
C(x, 0)=\bar{C} \delta(x)
$$

in the paper. ${ }^{1 \prime}$ It is incorrect in dimension. The mean concentration of $\bar{C}\left[\mathrm{~mol} \cdot \mathrm{m}^{-3}\right]$ in Eq. (1) should be replaced with moles per unit area $E=\bar{C} \cdot L[\mathrm{~mol}$. $\left.\mathrm{m}^{-2}\right],{ }^{2)}$ because $\delta(x)$ has the dimension of the reciprocal of length $\left[\mathrm{m}^{-1}\right]$. Then Eq. (1) is correctly expressed as

$$
C(x, 0)=E \delta(x)=f(x)
$$

Equations (1), (3) and (4) ${ }^{11}$ together with Eq. (2) are expressed in the form of a Laplace transform as

$$
\begin{gathered}
d^{2} Y / d x^{2}-\left(\bar{U} / D_{a}\right)(d Y / d x)-s Y / D_{a}=-f(x) / D_{a} \\
Y(0, s)=Y(L, s) \\
d Y(0, s) / d x=d Y(L, s) / d x
\end{gathered}
$$

Equation (3) is a second-order linear differential equation with constant coefficients. Then the general solution is given as

$$
\begin{aligned}
Y(x, s)= & {\left[C_{1}(s)-\int_{0}^{x} f(\xi) \exp \left(-r_{1} \xi\right) /\left\{D_{a}\left(r_{1}-r_{2}\right)\right\} d \xi\right] } \\
& \times \exp \left(r_{1} x\right) \\
& +\left[C_{2}(s)+\int_{0}^{x} f(\xi) \exp \left(-r_{2} \xi\right) /\left\{D_{a}\left(r_{1}-r_{2}\right)\right\} d \xi\right] \\
& \times \exp \left(r_{2} x\right)
\end{aligned}
$$

where

$$
\begin{aligned}
& r_{1}=\bar{U} / 2 D_{a}+\sqrt{\left(\bar{U} / 2 D_{a}\right)^{2}+s / D_{a}}, \\
& r_{2}=\bar{U} / 2 D_{a}-\sqrt{\left(\bar{U} / 2 D_{a}\right)^{2}+s / D_{a}} .
\end{aligned}
$$

Integral constants $C_{1}(s)$ and $C_{2}(s)$ in Eq. (6) are determined by the use of boundary conditions of Eqs. (4) and (5) as

$$
\begin{aligned}
C_{1}(s) & =-B_{1}(s) \exp \left(r_{1} L\right) /\left[1-\exp \left(r_{1} L\right)\right] \\
& =+B_{1}(s) \sum_{n=0}^{\infty} \exp \left(\underline{\left.-n r_{1} L\right)} ;\right. \\
B_{1}(s) & =\int_{0}^{L} f(\xi) \exp \left(-r_{1} \xi\right) /\left\{D_{a}\left(r_{1}-r_{2}\right)\right\} d \xi \\
C_{2}(s) & =+B_{2}(s) \exp \left(r_{2} L\right) /\left[1-\exp \left(r_{2} L\right)\right] \\
& =+B_{2}(s) \sum_{n=1}^{\infty} \exp \left(+n r_{2} L\right) ; \\
B_{2}(s) & =\int_{0}^{L} f(\xi) \exp \left(-r_{2} \xi\right) /\left\{D_{a}\left(r_{1}-r_{2}\right)\right\} d \xi
\end{aligned}
$$

When one determines $C_{1}(s)$ and $C_{2}(s)$, he must skillfully expand them in power series so as to make the signs of root terms in $r_{1}$ and $r_{2}$ negative, as shown by the underlines in Eqs. (7) and (8). In this way, the inverse Laplace transform of Eq. (6) is performable.

Substituting Eqs. (7) and (8) and $f(\xi)=E \delta(\xi)$ into Eq. (6), it is rearranged to

$$
\begin{aligned}
Y(x, s)= & E\left[\sum_{n=1}^{\infty} \exp \left\{-r_{1}(n L-x)\right\}\right. \\
& \left.+\sum_{n=0}^{\infty} \exp \left\{+r_{2}(n L+x)\right\}\right]\left\{D_{a}\left(r_{1}-r_{2}\right)\right\}
\end{aligned}
$$

Substitute $r_{1}$ and $r_{2}$ into Eq. (9) and move the image-space by $-\left(\bar{U}^{2} / 4 D_{a}\right)$ through multiplying the original function $C(x, t)$ by $\exp \left(\bar{U}^{2} / 4 D_{a}\right)$. Then perform the inverse Laplace transform by use of the wellknown formula $\exp (-k \sqrt{s}) / \sqrt{s} \rightarrow \exp \left(-k^{2} / 4 t\right)$ 
$\sqrt{\pi t} ; k>0$. Thus is derived the exact form of Eq. (5) in the paper. ${ }^{1}$

At that time $C_{1}(s)$ was expanded in power series as

$$
\begin{aligned}
C_{1}(s) & =-B_{1}(s) \exp \left(r_{1} L\right) /\left[1-\exp \left(r_{1} L\right)\right] \\
& =-B_{1}(s) \sum_{n=1}^{\infty} \exp \left(+n r_{1} L\right)
\end{aligned}
$$

in the same form as $C_{2}(s)$. Then the inverse Laplace transform was not successfully performed and the exact solution could not be derived in a complete form.

Care is necessary not to fall in "a trap" of expansion in power series.

\section{Nomenclature}

$C(x, t)$

$=$ concentration

$\left[\mathrm{mol} \cdot \mathrm{m}^{-3}\right]$

$D_{a} \quad=$ longitudinal dispersion coefficient

$$
\begin{array}{llr}
E & =\text { moles of tracer pulse per unit area } & {\left[\mathrm{mol} \cdot \mathrm{m}^{-2}\right]} \\
k & =\text { constant greater than zero } & {[-]} \\
L & =\text { total length of loop reactor } & {[\mathrm{m}]} \\
n & =\text { integer variable number } & {[-]} \\
s & =\text { Laplace parameter } & {[-]} \\
t & =\text { time } & {\left[\mathrm{m} \cdot \mathrm{s}^{-1}\right]} \\
\bar{U} & =\text { average circulating velocity } & {\left[\mathrm{mol} \cdot \mathrm{m}^{-3}\right]} \\
Y(x, s) & =\text { Laplace transform of concentration } & {[\mathrm{m}]} \\
x & =\text { axial position of loop reactor } & \\
\delta(x) & =\text { delta function } & \\
\langle\text { Superscript }\rangle &
\end{array}
$$$$
\left[\mathrm{mol} \cdot \mathrm{m}^{-2}\right]
$$$$
[-]
$$$$
[\mathrm{m}]
$$$$
[-]
$$$$
[-]
$$

\section{Literature Cited}

1) Murakami, Y., T. Hirose, S. Ono and T. Nishijima: J. Chem. Eng. Japan, 15, 121 (1982).

2) Takao, M., S. Ono, T. Hirose and Y. Murakami: Chem. Eng. Sci., 37, 796 (1982). 